\title{
Impact of glycaemic index and dietary fibre on insulin sensitivity during the refeeding phase of a weight cycle in young healthy men
}

\author{
Merit Lagerpusch ${ }^{1}$, Janna Enderle ${ }^{1}$, Wiebke Later ${ }^{1}$, Ben Eggeling ${ }^{1}$, Detlef Pape ${ }^{2}$, Manfred J. Müller ${ }^{1}$ \\ and Anja Bosy-Westphal ${ }^{1,3 *}$ \\ ${ }^{1}$ Institute of Human Nutrition and Food Science, Christian-Albrechts University, Kiel, Germany \\ ${ }^{2}$ InsuLean GmbH \& Co KG, Essen, Germany \\ ${ }^{3}$ Institute of Nutritional Medicine, University of Hohenheim, Frubwirthstraße 12, 70593 Stuttgart, Germany
}

(Submitted 21 May 2012 - Final revision received 6 September 2012 - Accepted 6 September 2012 - First published online 29 November 2012)

\begin{abstract}
Previous studies suggest that a low-glycaemic index (LGI) diet may improve insulin sensitivity (IS). As IS has been shown to decrease during refeeding, we hypothesised that an LGI- $v$. high-GI (HGI) diet might have favourable effects during this phase. In a controlled nutritional intervention study, sixteen healthy men (aged $26 \cdot 8$ (SD $4 \cdot 1$ ) years, BMI $23 \cdot 0(\mathrm{SD} 1 \cdot 7) \mathrm{kg} / \mathrm{m}^{2}$ ) followed 1 week of overfeeding, 3 weeks of energy restriction and of 2 weeks refeeding at $\pm 50 \%$ energy requirement ( $50 \%$ carbohydrates, $35 \%$ fat and $15 \%$ protein). During refeeding, subjects were divided into two matched groups receiving either high-fibre LGI or lower-fibre HGI foods (GI $40 v$. 74 , fibre intake $65(\mathrm{SD} 6) v .27(\mathrm{SD} 4) \mathrm{g} / \mathrm{d}$ ). Body weight was equally regained in both groups with refeeding (mean regain 70.5 (SD 28.0 ) \% of loss). IS was improved by energy restriction and decreased with refeeding. The decreases in IS were greater in the HGI than in the LGI group (group $\times$ time interactions for insulin, homeostasis model assessment of insulin resistance $\left(\mathrm{HOMA}_{\mathrm{IR}}\right)$, Matsuda IS index $\left(\mathrm{Matsuda}_{\mathrm{ISI}}\right)$; all $P<0 \cdot 05)$. Mean interstitial glucose profiles during the day were also higher in the HGI group $\left(\Delta \mathrm{AUC} \mathrm{HGI}_{\mathrm{HGI}}\right.$ of continuous interstitial glucose monitoring: $6.6 \mathrm{mmol} / 1$ per $14 \mathrm{~h}, P=0.04)$. At the end of refeeding, parameters of IS did not differ from baseline values in either diet group (adiponectin, insulin, $\mathrm{HOMA}_{\mathrm{IR}}$, Matsuda $\mathrm{ISI}_{\mathrm{I}}, M$-value; all $P>0.05$ ). In conclusion, nutritional stress imposed by dietary restriction and refeeding reveals a GI/fibre effect in healthy non-obese subjects. LGI foods rich in fibre may improve glucose metabolism during the vulnerable refeeding phase of a weight cycle.
\end{abstract}

Key words: Glycaemic index: Refeeding: Insulin sensitivity: Weight cycle

Both the quantity and the type of carbohydrates in a food have been proposed to influence insulin sensitivity (IS). Jenkins et $a l^{(1)}$ initially developed the concept of glycaemic index (GI), stating that carbohydrate-containing foods that are either slowly or rapidly absorbed lead to different glycaemic responses. Repeated cycles of early postprandial hyperglycaemia and hyperinsulinaemia are characteristic of high-GI (HGI) diets and may cause impaired IS and $\beta$-cell dysfunction ${ }^{(2)}$. Accordingly, some prospective observational studies have revealed a positive association between GI and the risk of type 2 diabetes $^{(3,4)}$. Likewise, a low-GI (LGI) diet has repeatedly been shown to improve carbohydrate metabolism in a variety of intervention studies lasting $2-52$ weeks $^{(5-9)}$. However, these studies mainly included 'at risk' populations, i.e. overweight, obese, diabetic or hyperlipidaemic participants or those with CHD. It has been hypothesised that patients with high fasting and postprandial glucose concentrations may be more responsive to the effects of an LGI diet than healthy euglycaemic subjects ${ }^{(10)}$.

The effect of LGI $v$. HGI diets on IS in healthy normalweight subjects is less clear ${ }^{(7,10)}$. Several dietary intervention studies in healthy lean individuals have shown improvements in carbohydrate metabolism with LGI compared with HGI $\operatorname{diets}^{(8,11,12)}$. By contrast, IS assessed by euglycaemic clamp was impaired after 4 weeks of an LGI diet in lean men ${ }^{(13)}$. Other studies have failed to find a difference in IS between LGI and HGI diets in healthy non-obese subjects ${ }^{(14-16)}$. Importantly, the majority of previous studies have focused on modulation of GI during eu-energetic feeding or are constrained by a lack of controlled feeding conditions.

An issue of particular interest is the GI effect during nutritional stress imposed by dietary restriction followed by

Abbreviations: CGM, continuous glucose monitor; EI, energy intake; GI, glycaemic index; HGI, high glycaemic index; HOMA assessment of insulin resistance; iAUC, incremental AUC; IS, insulin sensitivity; LGI, low glycaemic index; Matsuda ${ }_{\mathrm{ISI}}$, Matsuda insulin sensitivity index; OGTT, oral glucose tolerance test.

*Corresponding author: Professor Anja Bosy-Westphal, fax + 49431 8805679, email anja.bosy-westphal@uni-hohenheim.de 
a refeeding period. Perturbations in energy balance and associated body weight fluctuations are common in normal to overweight individuals, as shown by holiday ${ }^{(17)}$ and weekend weight gain $^{(18)}$ as well as weight gain during college years $^{(19)}$. This weight gain is often followed by weight loss, resulting in repeated weight cycles. It is of great interest to study early metabolic alterations that could precede the development of obesity and obesity-related conditions, such as impaired glucose metabolism, to study potential preventive strategies. Recently, we have shown that 1 week of refeeding following 1 week of energy restriction reduced fasting and postprandial IS and increased glucose-stimulated insulin secretion in a group of healthy lean men ${ }^{(20)}$. The observed characteristics during refeeding may be explained by a sudden shift from fat to carbohydrate metabolism ${ }^{(21)}$. Refeeding an LGI diet after weight loss may therefore counteract the metabolic changes by reducing postprandial glucose and insulin concentrations and increasing serum adiponectin concentrations $^{(22,23)}$. Already slightly elevated postprandial glucose values have been proposed to be associated with an increased cardiac risk ${ }^{(24)}$.

To the best of our knowledge, the present study is the first that examines the impact of GI on IS during controlled refeeding. In a group of young healthy men, we compared the effects of an LGI $v$. HGI refeeding diet by applying continuous interstitial glucose monitoring as well as basal state (fasting glucose, insulin and adiponectin, homeostasis model assessment of insulin resistance $\left(\mathrm{HOMA}_{\mathrm{IR}}\right)$, steady state (hyperinsulinaemic euglycaemic clamp) and dynamic techniques to assess IS (oral glucose tolerance test, OGTT). We hypothesised that (1) an LGI $v$. HGI diet during refeeding would result in an improved IS via attenuated glycaemia and insulinaemia when compared with the preceding energy restriction period and (2) that an LGI $v$. HGI diet would have no effect on IS after restoration of energy balance.

\section{Subjects and methods}

\section{Subjects}

A total of sixteen healthy men aged 22-37 years were recruited by notice board postings and mailing lists from the University of Kiel campus. Health status was assessed by medical history and physical examination. All subjects were normal weight, except for three participants, whose BMI ranged between 25.6 and $26.2 \mathrm{~kg} / \mathrm{m}^{2}$. Subjects eligible for participation were non-smokers, weight stable ( $\pm 2 \mathrm{~kg})$ within the preceding 12 months, did not use any medications, had no family history of type 2 diabetes, no food allergies and did not follow any special diets (e.g. vegetarian). Competitive athletes were also excluded from the study and subjects were instructed to refrain from exercise in the 1 week pre-intervention period. The present study was conducted according to the guidelines laid down in the Declaration of Helsinki, and all procedures involving human subjects were approved by the ethics committee of the Medical Faculty of the Christian-Albrechts-University of Kiel. Written informed consent was obtained from all subjects.

\section{Experimental protocol}

The 6-week controlled nutritional intervention study comprised 1 week of overfeeding $(+50 \%$ of energy requirement), 3 weeks of energy restriction $(-50 \%$ of energy requirement) and 2 weeks of refeeding $(+50 \%$ of energy requirement). During refeeding, subjects were divided into two sub-groups ( $n$ 8) receiving either high-fibre LGI or HGI foods. The subgroups were matched according to age, body weight, percentage body fat and glucose tolerance status (as assessed by OGTT). The initial overfeeding period was added to the study protocol to limit minimum body weight.

Before the dietary intervention, there was a 1-week preintervention period, during which each subject underwent a hyperinsulinaemic-euglycaemic clamp and an OGTT. Prior to the tests, resting energy expenditure was measured to determine individual energy requirement. Body weight and blood pressure were measured daily throughout the 6-week dietary intervention. Measurements of resting energy expenditure and OGTT as well as fasting blood sampling were repeated at the end of energy restriction and refeeding. Continuous interstitial glucose monitoring and a second euglycaemic clamp were performed at the end of the refeeding period. An outline of the experimental protocol is shown in Fig. 1.

Participants arrived at the metabolic ward of the Institute of Human Nutrition every morning at 08.30 hours after an

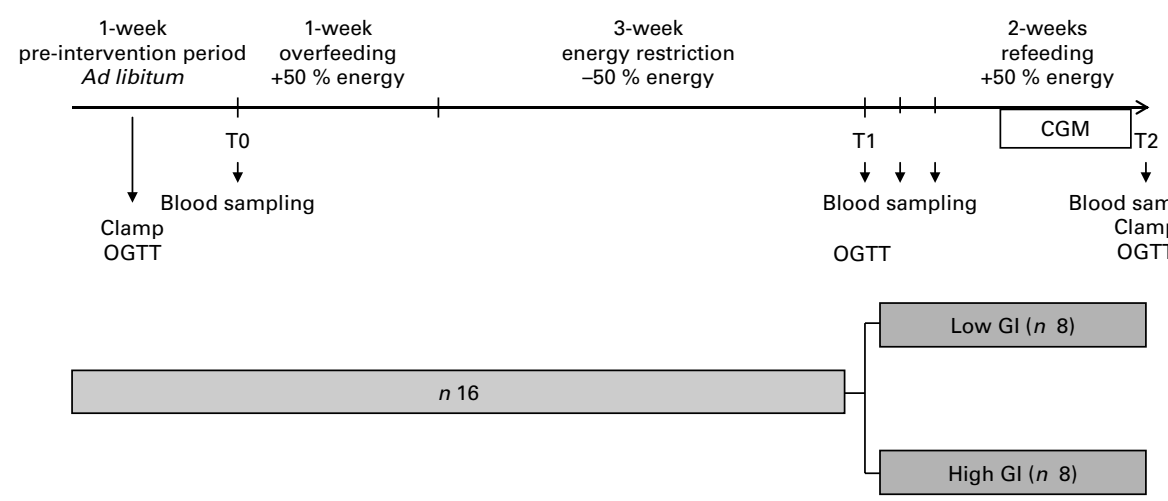

Fig. 1. Schematic overview of the study protocol. OGTT, oral glucose tolerance test; CGM, continuous glucose monitoring; GI, glycaemic index; T0, baseline; $\mathrm{T} 1$, end of energy restriction; T2, end of refeeding. 
overnight fast of $\geq 10 \mathrm{~h}$. During the pre-intervention period, they were allowed to leave the institute after all measurements were completed. Throughout the intervention period, subjects were requested to stay at the institute until dinner.

\section{Experimental diet}

During the pre-intervention period, habitual ad libitum food intake was allowed and assessed by $3 \mathrm{~d}$ food records. Energy intake (EI) was increased by $50 \%$ of energy requirements during overfeeding and refeeding (mean EI 16701 (SD 1918) kJ/d), and was reduced by $50 \%$ during energy restriction (mean EI 5568 (SD 641) kJ/d). Individual energy requirement was calculated by multiplying resting energy expenditure by a physical activity level of $1 \cdot 4$, corresponding to sedentary behaviour during the entire study duration. A physical activity level of 1.4 was maintained by limiting physical activity to approximately 5000 steps/d. This was controlled by wearing activity monitors (SenseWear Pro3 Armband, Body-Media, Inc.). Subjects were also instructed to refrain from exercise throughout the entire study.

During the initial overfeeding period, subjects received a normal mixed diet. To standardise dietary intake, $50 \%$ of the EI during energy restriction and LGI $v$. HGI refeeding was given as a liquid formula diet that was provided free of charge by the InsuLean company (InsuLean $\mathrm{GmbH}$ \& Company KG). During energy restriction, the subjects consumed two liquid formula meals per $\mathrm{d}$ that were prepared with soya milk and enriched with lactose (18.9 (SD 2.0) g/drink) and sucrose (14.1 (SD 1.5) g/drink). During HGI refeeding, two liquid formula meals were prepared with lactose-free milk and two servings of grape juice were enriched with maltodextrin (43.1 (SD 6.4) g/drink). During LGI refeeding, three liquid formula meals prepared with soya milk and enriched with lactose $(42 \cdot 7$ (SD 2.2) g/drink) and sucrose (32.0 (SD 1.6) g/drink) were consumed. Because some subjects reported diarrhoea and flatulence on the LGI diet, $50 \%$ of lactose was replaced by sucrose in the first week of refeeding (lactose 21.3 (SD 1.1) g/drink, sucrose 53.3 (SD 2.7$) \mathrm{g} /$ drink). The remaining $50 \%$ of energy was provided as HGI and LGI mixed-meals and snacks, respectively. Loweror higher-GI versions of key 'staple' carbohydrate-rich foods were incorporated into the diet (e.g. lower- or higher-GI breads, rice, pasta and potato products). The relative glycaemic responses to the formula meals consumed during refeeding are given in the sample diets that are provided as Supplementary information (available online).

All foods and beverages consumed were provided during the intervention. The $7 \mathrm{~d}$ cycle menus were prepared by an experienced dietitian by using PRODI $^{\circledR}$ software (NutriScience $\mathrm{GmbH}$ ). The composition of the refeeding diet is given in Table 1. GI values of individual foods were taken from published international tables ${ }^{(25)}$. The GI of the diet was calculated according to WHO/FAO guidelines ${ }^{(26)}$ and averaged 40 units in the LGI and 74 units in the HGI diet, respectively. All diets consisted of $50 \%$ of total daily energy as carbohydrate, $15 \%$ as protein and $35 \%$ as fat. Saturated, monounsaturated and polyunsaturated fats accounted for
Table 1. Composition of the high-glycaemic index (HGI) and low-GI (LGI) refeeding diets

(Mean values and standard deviations)

\begin{tabular}{lccccc}
\hline & \multicolumn{2}{c}{ HGI } & & \multicolumn{2}{c}{ LGI } \\
\cline { 2 - 3 } Constituent & Mean & SD & & Mean & SD \\
\hline Carbohydrate (\% of total energy) & 50 & - & 50 & - \\
Protein (\% of total energy) & 15 & - & 15 & - \\
Fat (\% of total energy) & 35 & - & 35 & - \\
$\quad$ Saturated & 19 & - & 14 & - \\
$\quad$ Monounsaturated & 9 & - & 11 & - \\
$\quad$ Polyunsaturated & 2 & - & 5 & - \\
Fibre (g/d) & & & & \\
$\quad$ Total & 27 & 4 & 65 & 6 \\
$\quad$ Soluble & 11 & 1 & & 27 & 2 \\
$\quad$ Insoluble & 16 & 2 & 38 & 4 \\
Gl & 74 & 3 & & 40 & 3 \\
\hline
\end{tabular}

19, 9 and $2 \%$ of the total energy in the HGI diet and for 14, 11 and $5 \%$ of the total energy in the LGI diet, respectively. Refeeding diets were not matched for fibre content, which was 65 (SD 6) g/d (27 (SD 2) g soluble fibre and 38 (SD 4) g insoluble fibre) in the LGI and 27 (SD 4) g/d (11 (SD 1) g soluble fibre and 16 (SD 2) $\mathrm{g}$ insoluble fibre) in the HGI diet group $(P<0 \cdot 001)$. Subjects were instructed to eat all the foods provided, and meal intake was supervised by a skilled nutritionist. Participants were allowed to drink water, decaffeinated coffee and tea $a d$ libitum and the fluid intake was recorded.

\section{Anthropometric measurements and body composition analysis}

Height was measured to the nearest $0.5 \mathrm{~cm}$ using a stadiometer, with subjects not wearing shoes. Body weight was measured to the nearest $0.05 \mathrm{~kg}$ on an electronic scale (Seca 285, Seca GmbH \& Co KG) with subjects in underwear and after voiding. Waist circumference was measured to the nearest $0.5 \mathrm{~cm}$ midway between the lowest rib and the iliac crest. Fat-free mass was assessed by air-displacement plethysmography (BOD-POD Body Composition System, Life Measurement Instruments), as described in detail elsewhere ${ }^{(27)}$.

\section{Energy expenditure}

Respiratory exchange measurements were performed by means of an open-circuit indirect calorimeter (ventilated hood system Vmax 29n, SensorMedics ${ }^{\circledR}$; Sensor Medics 130 $\mathrm{GmbH}$ ), as previously described ${ }^{(28)}$. Oxygen consumption and carbon dioxide production were continuously measured for $\geq 30 \mathrm{~min}$ and resting energy expenditure was calculated from steady-state intervals using a standard formula ${ }^{(29)}$. Activity energy expenditure was assessed by the SenseWear Pro3 Armband (Body-Media, Inc.) that was continuously worn on the subject's dominant arm.

\section{Blood sampling and analytic procedures}

Plasma glucose was measured immediately upon sampling during hyperinsulinaemic-euglycaemic clamp and OGTT 
using a glucose oxidase method (BIOSEN C-Line, EKFdiagnostic). Fasting blood samples were obtained from an antecubital vein at the beginning of the study and at the end of energy restriction and refeeding. Fasting insulin and adiponectin concentrations were additionally analysed on days 2 and 4 of refeeding. After centrifugation for $10 \mathrm{~min}$ at $2000 \mathrm{~g}$ and $20^{\circ} \mathrm{C}$ within $40 \mathrm{~min}$ of sampling, the supernatant was stored at $-40^{\circ} \mathrm{C}$ until assayed. Serum insulin was determined by electrochemiluminescence immunoassay (Elecsys ${ }^{\circledR}$, Roche Diagnostics). Serum adiponectin was analysed by ELISA (ELISA E09, Mediagnost).

\section{Hyperinsulinaemic-euglycaemic clamp}

During the pre-intervention period and at the end of refeeding, peripheral IS was assessed by the hyperinsulinaemiceuglycaemic clamp technique, as previously described ${ }^{(20,30)}$. Whole-body IS ( $M$-value, expressed as $\mathrm{mg} / \mathrm{kg}$ fat-free mass per min) was determined from the mean rate of exogenous glucose infusion during the last $20 \mathrm{~min}$ of the clamp after correction for changes in the glucose pool size. Steady-state concentrations of serum insulin and plasma glucose $\left(\mathrm{CV}_{\mathrm{T} 0}\right.$ 5.8 (SD 2.3)\%) were assayed using previously described methods ${ }^{(30)}$.

\section{Oral glucose tolerance test}

A standard $75 \mathrm{~g}$ OGTT was performed during the preintervention period and at the end of energy restriction and refeeding with venous blood sampling for glucose and insulin determinations at 0, 30, 60, 90, 120 and $180 \mathrm{~min}$. Subjects lay quietly in a semi-recumbent position while watching video movies throughout the test. Glucose and insulin responses were calculated as incremental AUC (iAUC) above baseline using the trapezoidal method ${ }^{(31)}$. Missing data were due to not being able to collect blood samples.

\section{Continuous glucose monitoring}

In a sub-group of subjects ( $n 4$ in each GI group), interstitial glucose concentrations were measured by means of the FreeStyle Navigator ${ }^{\circledR}$ continuous glucose monitoring (CGM) device (Abbott Diabetes Care) at the end of the refeeding period. A glucose oxidase-based sensor was applied to the back of the upper arm to measure extracellular fluid glucose in subcutaneous tissue for five consecutive days. Sensor readings were reported every minute and an average value was stored in the monitor at every $10 \mathrm{~min}$. Subjects were instructed on how to calibrate the device based on five separate finger stick capillary blood samples collected during this period. Total AUC for nighttime (total AUC-CGM night: $_{\text {: }}$ : midnight to 07.00 hours) and iAUC for daytime (iAUCCGM $_{\text {day }}$ : 08.00-22.00 hours) glucose were calculated using the trapezoidal method ${ }^{(31)}$. Although sensor readings were recorded for the whole $5 \mathrm{~d}$ period, only the $24 \mathrm{~h}$ values monitored on Friday during the second week of refeeding were used for analysis.

\section{Parameters of insulin sensitivity and insulin secretion}

To estimate IS and insulin secretion, previously validated indices from either fasting or OGTT-derived glucose and insulin concentrations were calculated. An estimate of fasting IS was obtained by $\mathrm{HOMA}_{\mathrm{IR}}$ : fasting glucose $\times$ fasting insulin/ $22 \cdot 5^{(32,33)}$. Postprandial IS was estimated by Matsuda wholebody IS index (Matsuda $\left.{ }_{\text {ISI }}\right): 10000 /(($ fasting glucose $\times$ fasting insulin $) \times($ mean glucose $\times$ mean insulin during OGTT $))^{1 / 2(34)}$. Overall, glucose-stimulated insulin secretion during OGTT was calculated as iAUC-insulin/iAUC-glucose ${ }^{(35)}$.

\section{Statistical analyses}

Statistical analyses were performed using SPSS for Windows version 15.0 (SPSS, Inc.). Data normality was tested using the Kolmogorov-Smirnov test. Group differences at single time points were analysed by unpaired Student's $t$ tests Within-group changes following energy restriction were examined using paired Student's $t$ tests. Differences between groups with refeeding, when compared with energy restriction, were analysed by a mixed-design ANOVA testing for differences between diet groups (main effect of diet), changes over time (main effect of time) and differences in time course between groups (diet group $\times$ time interaction) followed by pairwise comparisons with Bonferroni adjustment. Homogeneity of variance and sphericity were tested using Levene's test and Mauchly's test, respectively. Significant or near-significant interactions were followed up with an unpaired Student's $t$ test at each time point to examine whether the effect of group depends on the time period. Because of significant between-group differences at baseline, hyperinsulinaemic clamp-derived $M$-values were also analysed using ANCOVA, with baseline values as a covariate and refeeding scores as the dependent variable. All statistical tests were two-sided and results were considered statistically significant if $P<0.05$. Results are presented as means with their standard deviations.

\section{Results}

\section{Subject characteristics at baseline}

Baseline characteristics of the subjects are summarised in Table 2. There were no significant differences in age, anthropometric variables, body composition, blood pressure and energy expenditure between the LGI and HGI groups $(P>0 \cdot 05)$.

\section{Changes in body weight during the intervention}

Cumulative body weight changes for the 6-week duration of the study are shown in Fig. 2. When compared with baseline, body weight was significantly reduced following energy restriction $(-4.3$ (SD 0.8$) \mathrm{kg}, P<0.001)$, with no significant difference between the LGI and the HGI groups $(-4.4$ (SD 0.8$) \quad v . \quad-4.1 \quad(\mathrm{SD} 0.9) \mathrm{kg}, \quad P=0.50)$. Following refeeding, body weight was regained in both groups $(P<0 \cdot 001)$, but the changes were not significantly different 
Table 2. Subject characteristics at baseline

(Mean values and standard deviations)

\begin{tabular}{|c|c|c|c|c|c|c|}
\hline & \multicolumn{2}{|c|}{ All subjects $(n 16)$} & \multicolumn{2}{|c|}{ LGI diet group ( $n$ 8) } & \multicolumn{2}{|c|}{ HGI diet group ( $n$ 8) } \\
\hline & Mean & SD & Mean & $\mathrm{SD}$ & Mean & SD \\
\hline Age (years) & $26 \cdot 8$ & 4.1 & $27 \cdot 6$ & $4 \cdot 2$ & $26 \cdot 0$ & $4 \cdot 1$ \\
\hline Height $(\mathrm{cm})$ & 181.9 & $7 \cdot 3$ & 184.0 & $5 \cdot 3$ & 179.9 & 7.9 \\
\hline Weight (kg) & $76 \cdot 0$ & $6 \cdot 6$ & $77 \cdot 2$ & 4.8 & $74 \cdot 2$ & $8 \cdot 2$ \\
\hline $\mathrm{BMI}\left(\mathrm{kg} / \mathrm{m}^{2}\right)$ & 23.0 & 1.7 & $22 \cdot \overline{8}$ & 1.6 & $23 \cdot 1$ & 1.8 \\
\hline Waist circumference $(\mathrm{cm})$ & $83 \cdot 1$ & $6 \cdot 7$ & $82 \cdot 7$ & $7 \cdot 7$ & 83.4 & $6 \cdot 2$ \\
\hline Fat mass $(\%)$ & $17 \cdot 3$ & $5 \cdot 8$ & $16 \cdot 9$ & $5 \cdot 7$ & $17 \cdot 6$ & $6 \cdot 3$ \\
\hline Fat-free mass $(\mathrm{kg})$ & $63 \cdot 0$ & 7.8 & $66 \cdot 3$ & $7 \cdot 6$ & $59 \cdot 7$ & $7 \cdot 0$ \\
\hline Diastolic blood pressure $(\mathrm{mmHg})$ & 81 & 6 & 82 & 6 & 81 & 6 \\
\hline Systolic blood pressure $(\mathrm{mmHg})$ & 122 & 11 & 123 & 14 & 122 & 9 \\
\hline REE (kJ/d) & 7955 & 913 & 8051 & 410 & 7854 & 1264 \\
\hline$A E E(k J / d)$ & 2361 & 1214 & 2173 & 737 & 2533 & 1557 \\
\hline
\end{tabular}

LGI, low glycaemic index; HGI, high glycaemic index; REE, resting energy expenditure; AEE, activity energy expenditure.

between subjects consuming the LGI and the HGI diets (LGI: 3.4 (SD 1.1) kg, HGI: $2 \cdot 5$ (SD 1.1) kg compared with energy restriction, $P=0 \cdot 12$ ).

\section{Continuous glucose monitoring}

Mean $24 \mathrm{~h}$ interstitial glucose profiles at the end of the refeeding period are shown in Fig. 3(a). The incremental daytime AUC-CGM was significantly higher in the HGI group, whereas the total nighttime AUC-CGM did not differ between the LGI and HGI groups (Fig. 3(b) and (c)).

\section{Parameters of insulin sensitivity during refeeding compared with energy restriction}

Fasting and OGTT-derived parameters of IS following energy restriction and during the refeeding period are shown in Tables 3 and 4 . There were no between-group differences in any outcome variable at the end of energy restriction (all $P>0.05)$.

Fasting parameters of insulin sensitivity. Fasting measures of plasma glucose, serum insulin, serum adiponectin as well as $\mathrm{HOMA}_{\mathrm{IR}}$ were significantly decreased by energy restriction (Table 3). After $14 \mathrm{~d}$ of refeeding, serum adiponectin significantly increased, whereas plasma glucose remained unchanged. ANOVA showed no group $\times$ time interactions. In addition, fasting insulin and $\mathrm{HOMA}_{\mathrm{IR}}$ were significantly increased at the end of refeeding. Greater increases in insulin concentrations and $\mathrm{HOMA}_{\mathrm{IR}}$ were observed in the HGI group (Table 4).

Fasting levels of insulin and adiponectin were also analysed on days 2 and 4 of refeeding. Paired comparisons revealed that fasting insulin was significantly elevated on day 2 (LGI: $+1.99(\mathrm{sD} 2.47) \mathrm{mU} / \mathrm{l}(+13.80 \quad(\mathrm{sD}$ 17.19) pmol/l), HGI: $+3.71(\mathrm{sD} 2.80) \mathrm{mU} / \mathrm{l}(+25.78(\mathrm{sD} 19.47) \mathrm{pmol} / \mathrm{l}), P=0.004)$ and on day 4 of refeeding (LGI: +3.87 (SD 2.75 ) $\mathrm{mU} / 1$ $(+26.91(\mathrm{SD} 19 \cdot 11) \mathrm{pmol} / \mathrm{l}), \quad \mathrm{HGI}: \quad+9 \cdot 12(\mathrm{sD}$ 9.78) $\mathrm{mU} / 1$ $(+63.37$ (sD 67.95) $\mathrm{pmol} / \mathrm{l}), P=0.017$ ). At the end of the refeeding period, basal insulin levels remained unchanged when compared with day 4 of refeeding. No group $\times$ time interactions were present. Serum adiponectin was unaltered on day 2 (LGI: -0.22 (SD 1.00) $\mu \mathrm{g} / \mathrm{ml}$, HGI: +0.02 (SD $0.53) \mu \mathrm{g} / \mathrm{ml}, P=1.00$ for paired comparison), but was significantly increased on day 4 of refeeding (LGI: +1.33 (SD $0.73) \mu \mathrm{g} / \mathrm{ml}$, HGI: +1.12 (sD 0.36) $\mu \mathrm{g} / \mathrm{ml}, P<0.001$ for paired comparison). On day 4 of refeeding, adiponectin concentrations were significantly higher than those at the end of the refeeding period $(P<0.001$ for paired comparison). These changes occurred independently of the diet group.

Oral glucose tolerance test-derived parameters of insulin sensitivity. During energy restriction, Matsuda ISI $_{\text {significantly }}$ improved and glucose-stimulated insulin secretion tended to decline, whereas glucose and insulin responses to oral glucose ingestion remained unchanged (Table 3). After refeeding, iAUC-glucose decreased and glucose-stimulated insulin secretion increased towards baseline values. No group effects were noted. iAUC-insulin was not altered by refeeding in either group. There was a significant interaction between diet group and time, with a refeeding-induced decline in Matsuda $_{\text {ISI }}$ in the HGI group only $(P=0 \cdot 005)$. By contrast,

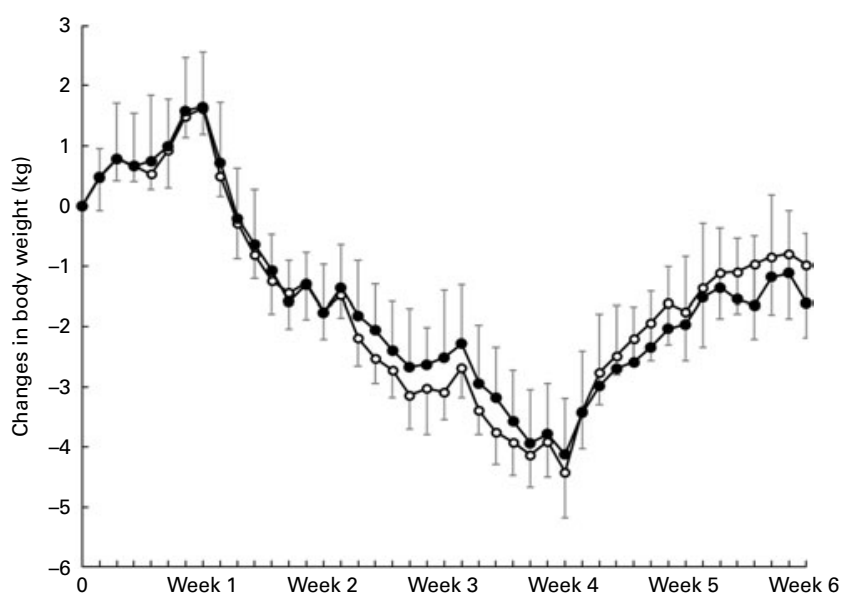

Fig. 2. Cumulative body weight changes for the 6-week duration of the study. Values are means, with standard deviations represented by vertical bars. -o-, Low-glycaemic index group ( $n$ 8); - - , high-glycaemic index group ( $n$ 8). 


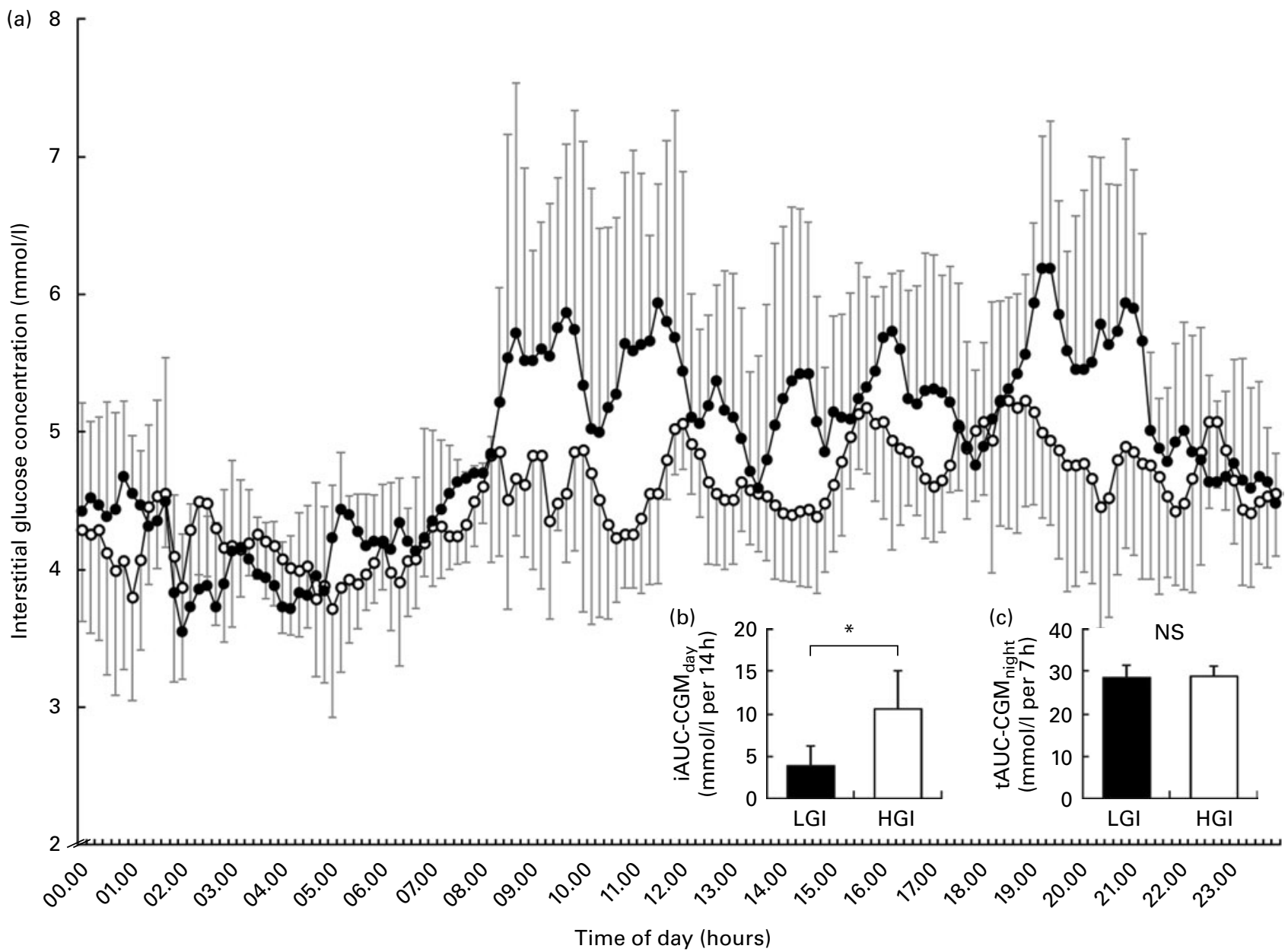

Fig. 3. Glycaemic responses over $24 \mathrm{~h}$ for the low-glycaemic index ( $\mathrm{LGI},-\mathrm{O}-$ ) and high-glycaemic index (HGI, $-\bullet$ ) diet groups. A subgroup of individuals ( $n$ 8) underwent continuous interstitial glucose monitoring (CGM) at the end of the LGI $(n 4)$ and $\mathrm{HGI}(n 4)$ refeeding period. (a) Interstitial glucose concentrations were measured every $10 \mathrm{~min}$ for $24 \mathrm{~h}$. Values are means, with standard deviations represented by vertical bars. (b) Incremental areas under the glucose-response curve during daytime (iAUC-CGM day, $08.00-22.00$ hours) and (c) total areas under the glucose-response curve during nighttime (tAUC-CGM ${ }_{\text {night }} 0-07.00$ hours) are shown as an insert. $\square, \mathrm{LGI} ; \mathbf{\square}, \mathrm{HGI} .{ }^{*}$ Mean values were significantly different between groups $(P=0.04)$.

refeeding had no effect on Matsuda $a_{\text {ISI }}$ in the LGI group $(P=0 \cdot 27)$ (Table 4).

\section{Parameters of insulin sensitivity at the end of refeeding compared with baseline}

Fasting-, OGTT- and clamp-derived parameters of IS at the end of refeeding compared with baseline are shown in Table 5. There were no significant baseline differences between the two diet groups, except for $M$-values that were significantly higher in the LGI group $(P=0.04)$.

Fasting parameters of insulin sensitivity. When compared with baseline, fasting glucose was significantly decreased and adiponectin levels tended to be elevated at the end of refeeding. No group $\times$ time interactions were observed. There were no significant changes in fasting insulin and $\mathrm{HOMA}_{\mathrm{IR}}$ in either group at the end of refeeding compared with baseline.

Oral glucose tolerance test-derived parameters of insulin sensitivity. No significant main effect of time or group $\times$ time interactions were observed with regard to AUC-glucose,
Table 3. Changes in parameters of insulin sensitivity (IS) from baseline (T0) to energy restriction (T1) in all subjects

(Mean values and standard deviations)

\begin{tabular}{|c|c|c|c|c|}
\hline & \multirow[b]{2}{*}{$n$} & \multicolumn{3}{|c|}{$\Delta \mathrm{T} 1-\mathrm{T} 0$} \\
\hline & & Mean & SD & $P^{*}$ \\
\hline \multicolumn{5}{|l|}{ Fasting parameters } \\
\hline Fasting glucose $(\mathrm{mmol} / \mathrm{l})$ & 16 & -0.23 & 0.23 & 0.001 \\
\hline Fasting insulin $(\mathrm{mU} / \mathrm{l}) \dagger$ & 16 & $-2 \cdot 46$ & $2 \cdot 74$ & 0.003 \\
\hline $\mathrm{HOMA}_{\mathrm{IR}}$ & 16 & -0.50 & 0.54 & 0.002 \\
\hline Fasting adiponectin $(\mu \mathrm{g} / \mathrm{ml})$ & 16 & -3.39 & 1.89 & $<0.001$ \\
\hline \multicolumn{5}{|l|}{ OGTT-derived parameters } \\
\hline iAUC-G (mmol/l per $3 \mathrm{~h})$ & 14 & 0.91 & $2 \cdot 33$ & $0 \cdot 17$ \\
\hline ¡AUC-I (mU/l per $3 \mathrm{~h}) \dagger$ & 14 & $-6 \cdot 37$ & 33.98 & 0.50 \\
\hline iAUC-I/iAUC-G & 14 & $-20 \cdot 25$ & $44 \cdot 30$ & $0 \cdot 11$ \\
\hline Matsuda $_{|S|}$ & 14 & 3.07 & 3.39 & 0.005 \\
\hline
\end{tabular}

$\mathrm{HOMA}_{\mathrm{IR}}$, homeostasis model assessment of insulin resistance; OGTT, oral glucose tolerance test; iAUC, incremental AUC; G, glucose; I, insulin; Matsuda IS, Matsuda insulin sensitivity index.

* Mean values at the end of energy restriction were significantly different from those at baseline (paired $t$ test).

† To convert insulin from mU// to pmol//, multiply by 6.945 . 
Table 4. Parameters of insulin sensitivity after energy restriction and after refeeding in the low-glycaemic index (LGI) and high-GI (HGl) diet groups

(Mean values and standard deviations)

\begin{tabular}{|c|c|c|c|c|c|c|c|c|}
\hline & \multirow[b]{2}{*}{$n^{*}$} & \multicolumn{2}{|c|}{ LGI group } & \multicolumn{2}{|c|}{ HGI group } & \multirow{2}{*}{$\begin{array}{c}\text { Time } \\
P \dagger\end{array}$} & \multirow{2}{*}{$\begin{array}{c}\text { Time } \times \text { diet group } \\
P \dagger\end{array}$} & \multirow{2}{*}{$\begin{array}{c}\text { Diet group } \\
P \dagger\end{array}$} \\
\hline & & Mean & SD & Mean & SD & & & \\
\hline \multicolumn{9}{|l|}{ Fasting parameters } \\
\hline Fasting glucose $(\mathrm{mmol} / \mathrm{l})$ & 8 & & & & & 0.72 & $0 \cdot 18$ & 0.77 \\
\hline $\mathrm{T} 1$ & & $4 \cdot 12$ & 0.23 & 4.04 & $0 \cdot 18$ & & & \\
\hline T2 & & 3.98 & 0.32 & $4 \cdot 12$ & 0.29 & & & \\
\hline$\Delta$ from $\mathrm{T} 1$ & & $-0 \cdot 14$ & 0.26 & 0.08 & 0.37 & & & \\
\hline Fasting insulin $(\mathrm{mU} / \mathrm{l}) \ddagger$ & 8 & & & & & $<0.001$ & 0.02 & 0.87 \\
\hline T1 & & 4.09 & 1.52 & $3 \cdot 16$ & 1.79 & & & \\
\hline T2 & & 5.49 & 1.25 & 6.69 & 2.69 & & & \\
\hline$\Delta$ from $\mathrm{T} 1$ & & 1.40 & 1.37 & 3.53 & $1 \cdot 19$ & & & \\
\hline $\mathrm{HOMA}_{\mathrm{IR}}$ & 8 & & & & & $<0.001$ & 0.02 & 0.87 \\
\hline $\mathrm{T} 1$ & & 0.76 & 0.31 & 0.58 & 0.34 & & & \\
\hline T2 & & 0.98 & 0.27 & 1.21 & 0.46 & & & \\
\hline$\Delta$ from $\mathrm{T} 1$ & & 0.22 & 0.29 & 0.63 & 0.35 & & & \\
\hline Fasting adiponectin $(\mu \mathrm{g} / \mathrm{ml})$ & 8 & & & & & $<0.001$ & 0.85 & 0.56 \\
\hline T1 & & 4.66 & 1.51 & 3.85 & $2 \cdot 66$ & & & \\
\hline T2 & & $9 \cdot 50$ & $3 \cdot 10$ & 9.08 & 3.89 & & & \\
\hline$\Delta$ from $\mathrm{T} 1$ & & 4.84 & $2 \cdot 67$ & $5 \cdot 24$ & $2 \cdot 72$ & & & \\
\hline \multicolumn{9}{|l|}{ OGTT-derived parameters } \\
\hline iAUC-G $(\mathrm{mmol} / \mathrm{l}$ per $3 \mathrm{~h})$ & 7 & & & & & 0.04 & 0.59 & 0.37 \\
\hline $\mathrm{T} 1$ & & $3 \cdot 71$ & 1.76 & 3.93 & 1.23 & & & \\
\hline T2 & & $2 \cdot 25$ & 0.92 & 3.04 & 1.55 & & & \\
\hline$\Delta$ from $\mathrm{T} 1$ & & -1.46 & 1.69 & -0.90 & $2 \cdot 13$ & & & \\
\hline iAUC-I (mU// per $3 \mathrm{~h}) \ddagger$ & 7 & & & & & 0.29 & 0.39 & 0.77 \\
\hline T1 & & 84.07 & 13.43 & 79.50 & 28.54 & & & \\
\hline T2 & & $86 \cdot 24$ & $46 \cdot 17$ & 99.62 & 36.45 & & & \\
\hline$\Delta$ from $\mathrm{T} 1$ & & $2 \cdot 17$ & 39.59 & $20 \cdot 12$ & 35.15 & & & \\
\hline iAUC-I/iAUC-G & 7 & & & & & 0.02 & 0.74 & 0.54 \\
\hline $\mathrm{T} 1$ & & $29 \cdot 38$ & $18 \cdot 60$ & 21.79 & $10 \cdot 81$ & & & \\
\hline $\mathrm{T} 2$ & & 43.52 & 26.98 & 39.99 & $20 \cdot 43$ & & & \\
\hline$\Delta$ from $\mathrm{T} 1$ & & $14 \cdot 13$ & 17.92 & $18 \cdot 21$ & $26 \cdot 34$ & & & \\
\hline Matsuda $_{|S|}$ & 7 & & & & & 0.001 & 0.02 & 0.68 \\
\hline $\mathrm{T} 1$ & & 11.62 & 3.84 & 14.65 & 5.47 & & & \\
\hline T2 & & $10 \cdot 34$ & 2.92 & 9.06 & 3.85 & & & \\
\hline$\Delta$ from $T 1$ & & $-1 \cdot 28$ & 2.79 & -5.59 & 3.40 & & & \\
\hline
\end{tabular}

$\mathrm{T} 1$, end of energy restriction; T2, end of refeeding; $\Delta$, change; $\mathrm{HOMA}_{\mathrm{IR}}$, homeostasis model assessment of insulin resistance; OGTT, oral glucose tolerance test; iAUC, incremental AUC; G, glucose; I, insulin; Matsuda ${ }_{I S I}$, Matsuda insulin sensitivity index.

${ }^{\star}$ Number of subjects in each group.

† Mixed-design ANOVA with time, diet group and time $\times$ diet group interaction as factors.

¥To convert insulin from $\mathrm{mU} / \mathrm{l}$ to $\mathrm{pmol} / \mathrm{l}$, multiply by 6.945 .

AUC-insulin, insulin secretion and Matsuda ${ }_{\text {ISI }}$ at the end of refeeding compared with baseline.

Clamp-derived parameters of insulin sensitivity. There were no changes over time in either group with respect to steady-state plasma glucose and steady-state serum insulin during the hyperinsulinaemic-euglycaemic clamp. Although $M$-values differed significantly between groups at baseline, ANCOVA provided no evidence of a covariate effect $(P=0.86)$ so that data were analysed using ANOVA. $M$-values during refeeding were unchanged from baseline and did not significantly differ between the LGI and HGI groups. Fat-free mass did not differ between groups following refeeding (LGI: 63.7 (sD 5.5) kg, HGI: 61.0 (sD 9.5) kg, $P=0.50$ ).

\section{Discussion}

In the present study, we investigated whether an LGI $v$. HGI diet may improve IS during refeeding in healthy, young, non-obese men. Interstitial glucose monitoring confirmed higher glucose responses during the day in the HGI diet group. When compared with the end of the energy restriction period, refeeding-induced decreases in fasting measures of IS were significantly greater in the HGI than in the high-fibre LGI group and OGTT-derived IS was decreased in the HGI group only. At the end of the refeeding period, fasting-, OGTT- and euglycaemic clamp-derived parameters of IS did not statistically differ from baseline values in either group.

\section{Effect of glycaemic index on daylong glycaemia}

Interstitial glucose measurements revealed higher glycaemia during the day on the HGI diet (Fig. 3). This shows that an LGI diet can be implemented under realistic conditions in terms of food choice and macronutrient composition to avoid excessive postprandial glucose excursions. By contrast, other authors reported no difference in the $24 \mathrm{~h}$ interstitial glucose concentrations in controlled laboratory or at home settings following $7 \mathrm{~d}$ macronutrient- and fibre-matched LGI and HGI diets in healthy overweight women ${ }^{(36)}$. The lower interstitial glucose concentrations on the LGI diet in the 
Table 5. Parameters of insulin sensitivity at baseline and after refeeding in the low-glycaemic index (LGI) and high-GI (HGI) diet groups (Mean values and standard deviations)

\begin{tabular}{|c|c|c|c|c|c|c|c|c|}
\hline & \multirow[b]{2}{*}{$n^{*}$} & \multicolumn{2}{|c|}{ LGI group } & \multicolumn{2}{|c|}{ HGI group } & \multirow{2}{*}{$\begin{array}{c}\text { Time } \\
P \dagger\end{array}$} & \multirow{2}{*}{$\begin{array}{c}\text { Time } \times \text { diet group } \\
P \dagger\end{array}$} & \multirow{2}{*}{$\begin{array}{c}\text { Diet group } \\
\qquad \dagger\end{array}$} \\
\hline & & Mean & SD & Mean & SD & & & \\
\hline \multicolumn{9}{|l|}{ Fasting parameters } \\
\hline Fasting glucose $(\mathrm{mmol} / \mathrm{l})$ & 8 & & & & & 0.009 & 0.90 & 0.20 \\
\hline T0 & & 4.25 & 0.18 & $4 \cdot 37$ & 0.22 & & & \\
\hline T2 & & 3.98 & 0.32 & $4 \cdot 12$ & 0.29 & & & \\
\hline$\Delta$ from $\mathrm{TO}$ & & -0.27 & 0.26 & -0.25 & 0.41 & & & \\
\hline Fasting insulin (mU/l)‡ & 8 & & & & & 1.00 & 0.52 & 0.46 \\
\hline T0 & & 5.99 & $2 \cdot 70$ & $6 \cdot 19$ & 2.50 & & & \\
\hline $\mathrm{T} 2$ & & 5.49 & $1 \cdot 25$ & 6.69 & 2.69 & & & \\
\hline$\Delta$ from T0 & & -0.50 & $2 \cdot 80$ & 0.50 & $3 \cdot 20$ & & & \\
\hline $\mathrm{HOMA}_{\mathrm{IR}}$ & 8 & & & & & 0.60 & 0.57 & 0.41 \\
\hline T0 & & $1 \cdot 14$ & 0.54 & $1 \cdot 21$ & 0.49 & & & \\
\hline T2 & & 0.98 & 0.27 & 1.21 & 0.46 & & & \\
\hline$\Delta$ from $\mathrm{TO}$ & & $-0 \cdot 16$ & 0.57 & 0.01 & 0.57 & & & \\
\hline Fasting adiponectin $(\mu \mathrm{g} / \mathrm{ml})$ & 8 & & & & & 0.05 & 0.29 & 0.40 \\
\hline T0 & & $8 \cdot 71$ & $3 \cdot 67$ & $6 \cdot 58$ & $2 \cdot 87$ & & & \\
\hline T2 & & $9 \cdot 50$ & $3 \cdot 10$ & 9.08 & 3.89 & & & \\
\hline$\Delta$ from T0 & & 0.78 & 3.84 & 2.49 & $2 \cdot 18$ & & & \\
\hline \multicolumn{9}{|l|}{ OGTT-derived parameters } \\
\hline iAUC-G (mmol// per $3 \mathrm{~h})$ & 7 & & & & & 0.58 & 0.20 & 0.86 \\
\hline T0 & & $3 \cdot 17$ & 1.96 & $2 \cdot 65$ & $2 \cdot 17$ & & & \\
\hline T2 & & $2 \cdot 25$ & 0.92 & 3.04 & 1.55 & & & \\
\hline$\Delta$ from T0 & & -0.92 & 1.76 & 0.38 & 1.81 & & & \\
\hline iAUC-I (mU// per $3 \mathrm{~h}) \ddagger$ & 7 & & & & & 0.75 & 0.53 & 0.78 \\
\hline T0 & & $90 \cdot 84$ & 40.98 & 85.47 & $6 \cdot 37$ & & & \\
\hline T2 & & $86 \cdot 24$ & $46 \cdot 17$ & 99.62 & 36.45 & & & \\
\hline$\Delta$ from T0 & & $-4 \cdot 60$ & 66.66 & 14.15 & 38.78 & & & \\
\hline iAUC-I/iAUC-G & 7 & & & & & 0.73 & 0.50 & 0.74 \\
\hline T0 & & $39 \cdot 60$ & $29 \cdot 70$ & 52.07 & 48.25 & & & \\
\hline T2 & & 43.52 & 26.98 & 39.99 & 20.43 & & & \\
\hline$\Delta$ from TO & & 3.92 & 34.93 & -12.07 & $50 \cdot 18$ & & & \\
\hline Matsuda $_{|S|}$ & 7 & & & & & 0.71 & 0.21 & 0.99 \\
\hline T0 & & 9.46 & 2.04 & $10 \cdot 67$ & $5 \cdot 23$ & & & \\
\hline T2 & & $10 \cdot 34$ & 2.92 & 9.06 & 3.85 & & & \\
\hline$\Delta$ from T0 & & 0.88 & 3.69 & -1.61 & 3.39 & & & \\
\hline \multicolumn{9}{|l|}{ Clamp-derived parameters } \\
\hline $\mathrm{SSPG}(\mathrm{mmol} / \mathrm{l})$ & 8 & & & & & 0.10 & 0.15 & 0.58 \\
\hline T0 & & 4.65 & 0.20 & 4.68 & 0.29 & & & \\
\hline T2 & & 4.63 & 0.11 & 4.50 & 0.21 & & & \\
\hline$\Delta$ from TO & & -0.01 & 0.20 & -0.18 & 0.23 & & & \\
\hline SSSI (mU/l) & 8 & & & & & 0.18 & 0.88 & 0.21 \\
\hline T0 & & $75 \cdot 39$ & 15.03 & $82 \cdot 13$ & $12 \cdot 14$ & & & \\
\hline T2 & & $79 \cdot 74$ & $46 \cdot 17$ & 87.50 & 36.45 & & & \\
\hline$\Delta$ from T0 & & 4.35 & 14.57 & $5 \cdot 37$ & 11.67 & & & \\
\hline$M$-value $\left(\mathrm{mg} / \mathrm{kg}_{\mathrm{FFM}}\right.$ per $\left.\mathrm{min}\right)$ & 8 & & & & & 0.87 & 0.12 & $0 \cdot 19$ \\
\hline T0 & & $11 \cdot 23$ & $2 \cdot 54$ & 8.64 & 1.80 & & & \\
\hline T2 & & 9.92 & $2 \cdot 35$ & $10 \cdot 24$ & $2 \cdot 26$ & & & \\
\hline$\Delta$ from T0 & & -1.31 & 3.56 & 1.60 & 2.94 & & & \\
\hline
\end{tabular}

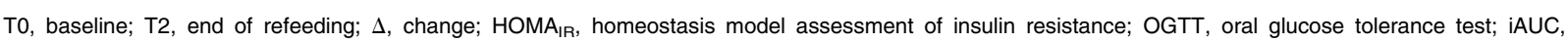
incremental AUC; G, glucose; I, insulin; Matsuda ${ }_{I S I}$, Matsuda insulin sensitivity index; SSPG, steady-state plasma glucose; SSSI, steady-state serum insulin. ${ }^{*}$ Number of subjects in each group.

$\dagger$ Mixed-design ANOVA with time, diet group and time $\times$ diet group interaction as factors.

$\ddagger$ To convert insulin from $\mathrm{mU} / \mathrm{l}$ to $\mathrm{pmol} / \mathrm{l}$, multiply by 6.945 .

present study could therefore be due to the lower GI of the diet as well as to the higher content of indigestible carbohydrates, which may have added to the beneficial effect on blood glucose over the course of the day. This is supported by the fact that the present results are in agreement with a previous study that showed a decrease in $24 \mathrm{~h}$ interstitial glucose after $7 \mathrm{~d}$ on a free-living LGI diet, with a concomitant increase in dietary fibre intake ${ }^{(37)}$. The combination of fibre and LGI has been shown to beneficially affect glycaemia at a subsequent meal through the 'second-meal' effect ${ }^{(19)}$.

\section{Parameters of insulin sensitivity during refeeding compared with energy restriction}

When compared with the end of the energy restriction period, the decreases in fasting measures of IS with refeeding were significantly greater in the HGI than in the LGI group (group $\times$ time interaction for serum insulin and $\mathrm{HOMA}_{\mathrm{IR}}$ ), and OGTTderived IS was decreased in the HGI group only (group $\times$ time interaction for Matsuda $\mathrm{ISI}_{\text {I }}$ ) (Table 4). The majority of previous GI interventions were designed as weight maintenance studies 
and did not find a beneficial effect of LGI diets on parameters of IS in healthy euglycaemic individuals ${ }^{(13-16)}$. By contrast, few controlled studies have investigated the effect of GI during periods of perturbed energy balance. These studies included type 2 diabetic $^{(38)}$, obese ${ }^{(39,40)}$, overweight ${ }^{(38,41)}$ and/or older individuals ${ }^{(40)}$ markedly improving their metabolic control by weight loss or exercise regimens, whereas our subjects were healthy and predominantly normal-weight at baseline. As opposed to the present findings, the earlier cited studies suggest that the GI effect is superimposed by weight loss or exercise regimens that exert a more pronounced effect on glucose metabolism and parameters of $\mathrm{IS}^{(38-41)}$. A 6-month energy-restricted LGI $v$. HGI diet $(-30 \% \mathrm{EI})$, which also differed in glycaemic load, equally improved fasting as well as OGTT-derived IS in overweight subjects, suggesting that GI does not affect glucose-insulin dynamics beyond that associated with weight changes ${ }^{(41)}$. The discrepant findings might not only be due to inclusion of different study populations, but also to differences in energy balance, because the participants of the present study were exposed to an energy surplus instead of a deficit. To our knowledge, there has been no study examining the GI effect in healthy normal-weight individuals during a positive energy balance. Subjects in the LGI group tended to regain slightly more body weight than those in the HGI group $(P=0 \cdot 12)$ (Fig. 2). Theoretical work suggests that insulin resistance prevents weight gain $^{(42,43)}$. It has been shown that an elevated IS was associated with the propensity of future weight gain ${ }^{(44)}$. A metabolic setting of increased IS by energy restriction has been related to altered partitioning of lipid fuels with accelerated uptake and storage in adipose tissue $^{(42)}$.

While energy restriction increased and refeeding decreased IS, adiponectin behaved inversely and decreased with energy restriction or increased with refeeding, respectively (Tables 2 and 3). This contrasts with the positive association between IS and serum adiponectin that is observed in cross-sectional studies $^{(45)}$. However, it has been suggested that a change in adiponectin is not a cause, but rather a consequence of long-term changes in IS $^{(46,47)}$

\section{Parameters of insulin sensitivity at the end of refeeding compared with baseline}

At the end of the refeeding period, fasting-, OGTT- and euglycaemic clamp-derived parameters of IS did not statistically differ from baseline values in either group (Table 4). This is in agreement with previous studies focusing on the effect of GI of the diet on IS during isoenergetic or ad libitum feeding ${ }^{(13-16)}$. Whereas a favourable effect of LGI has mainly been found in overweight subjects ${ }^{(8,9)}$ or those with cardiometabolic risk factors $^{(5-8)}$, healthy euglycaemic individuals might be less susceptible to changes in glucose-insulin dynamics in eu-energetic conditions (for reviews, see Jenkins et $a l .{ }^{(7)}$ and Livesey et $\left.a{ }^{(10)}\right)$. Results of a meta-analysis of GI interventions did not support an improved IS with LGI diets in healthy normalweight individuals, whereas beneficial effects were seen when fasting glucose levels exceeded $5 \mathrm{mmol} / \mathrm{l}$ or fasting insulin levels were above $100 \mathrm{pmol} / \mathrm{l}^{(10)}$. Accordingly, a controlled $8 \mathrm{~d}$ isoenergetic LGI $v$. HGI crossover study in young healthy lean adults revealed no group difference in glycaemic and insulinaemic responses to OGTT ${ }^{(14)}$. The present results are also in line with a study by Kiens \& Richter ${ }^{(13)}$ showing that fasting levels of glucose and insulin as well as whole-body glucose uptake at a low plasma insulin concentration during clamp were similar for isoenergetic LGI and HGI diets after 4 weeks in healthy young men. However, it may be speculated that a prolonged refeeding duration that results in an overfeeding situation with a positive cumulative energy balance could lead to a more pronounced difference in parameters of IS.

\section{Clinical importance and limitations of the findings}

Perturbations in energy balance and associated body weight fluctuations are common in normal-to-overweight individuals, as shown by weight gain over the weekend, holidays and during college years, for example ${ }^{(17-19)}$. This weight gain is often followed by weight loss, resulting in repeated weight cycles. As disease progress constitutes a continuum, it is of great interest to study early metabolic characteristics that are associated with these fluctuations in body weight and could precede the development of obesity and impaired glucose metabolism. The present results show that a high-fibre LGI diet may be preventive or may correct early metabolic changes in this group of healthy non-obese subjects under conditions of perturbed energy balance and presumably high insulin secretion. The clinical relevance of modestly reduced daylong glycaemia in the range of normal blood glucose levels is supported by the fact that postprandial glycaemia as low as $5.4 \mathrm{mmol} / \mathrm{l}$ has already been associated with an increased cardiac risk ${ }^{(24)}$.

As fibre content was significantly higher in the LGI diet, any difference in glycaemia and IS might also be ascribed to a higher intake of soluble and insoluble (mainly cereal) fibre. Soluble fibre has been shown to reduce glycaemia, whereas insoluble cereal fibre is associated with increased $\mathrm{IS}^{(48)}$. By contrast, other authors found no relationship between the consumption of whole-grain foods and IS in healthy subjects ${ }^{(49)}$. Moreover, although the LGI diet was considerably higher in dietary fibre, the HGI diet was also relatively high in fibre, with daily intakes approximating the recommended $30 \mathrm{~g}$ by the German Nutrition Society. It is difficult to create a diet differing in GI with equal fibre content when using commonly consumed food items, because LGI foods do generally have a higher content of indigestible carbohydrates. Moreover, larger differences in GI are difficult to achieve without increasing fibre intake ${ }^{(36)}$. The combination of a low GI and a high fibre intake has been shown to have the largest effect on disease risk reduction ${ }^{(10)}$. One reason may be the low GI of a high-fibre diet, but there may also be other aspects independent of GI, e.g. reduced nutrient absorption, altered secretion of gut hormones, a shift of the gut microbiota structure and modulation of inflammatory markers ${ }^{(48)}$. However, a lower nutrient absorption linked to a high fibre intake is highly unlikely in the present study, because subjects in the LGI group tended to regain more body weight than those in the HGI group. 
Although confounding effects of different fatty acid profiles in the two diets cannot be ruled out, it is likely that the present study protocol was too short and differences in fatty acid quality between diet groups were too small to detect significant effects on IS. Whereas a relationship between dietary fat quality and IS has been shown in a 3-month controlled trial, the majority of controlled and intervention studies in healthy subjects, particularly in the short term, have shown negative results ${ }^{(50)}$.

The present study has several strengths, including the strictly controlled nutrition regimen, monitoring of physical activity and the assessment of IS in the fasting state as well as under dynamic and steady-state conditions. Despite these strengths, the generalisability of the findings is limited by the relatively small sample size. However, effect sizes ranged from 0.31 to 0.54 and support the clinical significance of our findings.

\section{Conclusion}

In conclusion, the present study showed that refeeding caused a relative state of insulin resistance when compared with the preceding energy restriction period. The decrease in IS was more pronounced in the high-GI lower-dietary fibre group than in the low-GI high-dietary fibre group. A nutritional stress situation imposed by dietary restriction followed by refeeding may be necessary to evoke a GI/fibre effect in healthy non-obese individuals. Fibre-rich low-GI foods may improve glucose metabolism during a state of physiological vulnerability, i.e. the refeeding phase of a weight cycle or the weight maintenance phase after weight loss.

\section{Supplementary material}

To view supplementary material for this article, please visit http://dx.doi.org/10.1017/S000711451200462X

\section{Acknowledgements}

We thank Ulrike Preuß (Institute of Human Nutrition and Food Science, Christian-Albrechts University Kiel) for her assistance in food preparation and all the subjects for their participation in the study. The authors declare no conflict of interest. The present study was supported by a grant of the German Ministry of Education and Research (BMBF 0315681) and the German Research Foundation (DFG Bo 3296/1-1). M. J. M. and A. B.-W. designed the research; M. L., J. E., W. L., B. E. and A. B.-W. conducted the research; M. L., D. P., M. J. M. and A. B.-W. discussed the data; M. L. analysed the data; M. L., M. J. M. and A. B.-W. wrote the paper; M. L., A. B.-W. had primary responsibility for the final content. All authors read and approved the final manuscript.

\section{References}

1. Jenkins DJ, Wolever TM, Taylor RH, et al. (1981) Glycemic index of foods: a physiological basis for carbohydrate exchange. Am J Clin Nutr 34, 362-366.
2. Ludwig DS (2002) The glycemic index: physiological mechanisms relating to obesity, diabetes, and cardiovascular disease. JAMA 287, 2414-2423.

3. Salmeron J, Ascherio A, Rimm EB, et al. (1997) Dietary fiber, glycemic load, and risk of NIDDM in men. Diabetes Care 20, $545-550$

4. Schulze MB, Liu S, Rimm EB, et al. (2004) Glycemic index, glycemic load, and dietary fiber intake and incidence of type 2 diabetes in younger and middle-aged women. $A m \mathrm{~J}$ Clin Nutr 80, 348-356.

5. Wolever TM, Jenkins DJ, Vuksan V, et al. (1992) Beneficial effect of a low glycaemic index diet in type 2 diabetes. Diabet Med 9, 451-458.

6. Frost G, Keogh B, Smith D, et al. (1996) The effect of lowglycemic carbohydrate on insulin and glucose response in vivo and in vitro in patients with coronary heart disease. Metabolism 45, 669-672.

7. Jenkins DJ, Kendall CW, Augustin LS, et al. (2002) Glycemic index: overview of implications in health and disease. $A m J$ Clin Nutr 76, 266S-273S.

8. Brynes AE, Mark Edwards C, Ghatei MA, et al. (2003) A randomised four-intervention crossover study investigating the effect of carbohydrates on daytime profiles of insulin, glucose, non-esterified fatty acids and triacylglycerols in middle-aged men. Br J Nutr 89, 207-218.

9. Krog-Mikkelsen I, Sloth B, Dimitrov D, et al. (2011) A low glycemic index diet does not affect postprandial energy metabolism but decreases postprandial insulinaemia and increases fullness ratings in healthy women. $J$ Nutr 141, 1679-1684.

10. Livesey G, Taylor R, Hulshof $\mathrm{T}$, et al. (2008) Glycemic response and health - a systematic review and metaanalysis: relations between dietary glycemic properties and health outcomes. Am J Clin Nutr 87, 258S-268S.

11. Jenkins DJ, Wolever TM, Collier GR, et al. (1987) Metabolic effects of a low-glycemic-index diet. Am J Clin Nutr $\mathbf{4 6}$ 968-975.

12. Frost G, Leeds A, Trew G, et al. (1998) Insulin sensitivity in women at risk of coronary heart disease and the effect of a low glycemic diet. Metabolism 47, 1245-1251.

13. Kiens B \& Richter EA (1996) Types of carbohydrate in an ordinary diet affect insulin action and muscle substrates in humans. Am J Clin Nutr 63, 47-53.

14. Herrmann TS, Bean ML, Black TM, et al. (2001) High glycemic index carbohydrate diet alters the diurnal rhythm of leptin but not insulin concentrations. Exp Biol Med (Maywood) 226, 1037-1044.

15. Sloth B, Krog-Mikkelsen I, Flint A, et al. (2004) No difference in body weight decrease between a low-glycemic-index and a high-glycemic-index diet but reduced LDL cholesterol after 10-wk ad libitum intake of the low-glycemic-index diet. Am J Clin Nutr 80, 337-347.

16. Alfenas RC \& Mattes RD (2005) Influence of glycemic index/ load on glycemic response, appetite, and food intake in healthy humans. Diabetes Care 28, 2123-2129.

17. Yanovski JA, Yanovski SZ, Sovik KN, et al. (2000) A prospective study of holiday weight gain. $N$ Engl $J$ Med 342, $861-867$

18. Racette SB, Weiss EP, Schechtman KB, et al. (2008) Influence of weekend lifestyle patterns on body weight. Obesity (Silver Spring) 16, 1826-1830.

19. Crombie AP, Ilich JZ, Dutton GR, et al. (2009) The freshman weight gain phenomenon revisited. Nutr Rev 67, 83-94

20. Lagerpusch M, Bosy-Westphal A, Kehden B, et al. (2012) Effects of brief perturbations in energy balance on indices 
of glucose homeostasis in healthy lean men. Int $J$ Obes (Lond) 36, 1094-1101.

21. Crook MA, Hally V \& Panteli JV (2001) The importance of the refeeding syndrome. Nutrition 17, 632-637.

22. Pawlak DB, Kushner JA \& Ludwig DS (2004) Effects of dietary glycaemic index on adiposity, glucose homoeostasis, and plasma lipids in animals. Lancet 364, 778-785.

23. Neuhouser ML, Schwarz Y, Wang C, et al. (2012) A lowglycemic load diet reduces serum $\mathrm{C}$-reactive protein and modestly increases adiponectin in overweight and obese adults. J Nutr 142, 369-374.

24. Gerstein HC \& Yusuf S (1996) Dysglycaemia and risk of cardiovascular disease. Lancet 347, 949-950.

25. Foster-Powell K, Holt SH \& Brand-Miller JC (2002) International table of glycemic index and glycemic load values: 2002. Am J Clin Nutr 76, 5-56.

26. FAO/WHO (1998) Carbohydrates in human nutrition. Report of a Joint FAO/WHO Expert Consultation. FAO Food Nutr Pap 66, 1-140.

27. Bosy-Westphal A, Mast M, Eichhorn C, et al. (2003) Validation of air-displacement plethysmography for estimation of body fat mass in healthy elderly subjects. Eur J Nutr $\mathbf{4 2}$, 207-216

28. Bader N, Bosy-Westphal A, Dilba B, et al. (2005) Intra- and interindividual variability of resting energy expenditure in healthy male subjects - biological and methodological variability of resting energy expenditure. Br J Nutr 94, 843-849.

29. Weir JB (1949) New methods for calculating metabolic rate with special reference to protein metabolism. J Physiol 109, 1-9.

30. Bosy-Westphal A, Kossel E, Goele K, et al. (2010) Association of pericardial fat with liver fat and insulin sensitivity after diet-induced weight loss in overweight women. Obesity (Silver Spring) 18, 2111-2117.

31. Matthews JN, Altman DG, Campbell MJ, et al. (1990) Analysis of serial measurements in medical research. BMJ 300, $230-235$

32. Matthews DR, Hosker JP, Rudenski AS, et al. (1985) Homeostasis model assessment: insulin resistance and beta-cell function from fasting plasma glucose and insulin concentrations in man. Diabetologia 28, 412-419.

33. Hoffman RP (2008) Indices of insulin action calculated from fasting glucose and insulin reflect hepatic, not peripheral, insulin sensitivity in African-American and Caucasian adolescents. Pediatr Diabetes 9, 57-61.

34. Matsuda M \& DeFronzo RA (1999) Insulin sensitivity indices obtained from oral glucose tolerance testing: comparison with the euglycemic insulin clamp. Diabetes Care 22, $1462-1470$.

35. Retnakaran R, Shen S, Hanley AJ, et al. (2008) Hyperbolic relationship between insulin secretion and sensitivity on oral glucose tolerance test. Obesity (Silver Spring) 16, 1901-1907.
36. Aston LM, Laccetti R, Mander AP, et al. (2010) No difference in the 24-hour interstitial fluid glucose profile with modulations to the glycemic index of the diet. Nutrition $\mathbf{2 6}$, 290-295.

37. Brynes AE, Adamson J, Dornhorst A, et al. (2005) The beneficial effect of a diet with low glycaemic index on $24 \mathrm{~h}$ glucose profiles in healthy young people as assessed by continuous glucose monitoring. Br J Nutr 93, 179-182.

38. Heilbronn LK, Noakes M \& Clifton PM (2002) The effect of high- and low-glycemic index energy restricted diets on plasma lipid and glucose profiles in type 2 diabetic subjects with varying glycemic control. J Am Coll Nutr 21, 120-127.

39. Raatz SK, Torkelson CJ, Redmon JB, et al. (2005) Reduced glycemic index and glycemic load diets do not increase the effects of energy restriction on weight loss and insulin sensitivity in obese men and women. J Nutr 135, 2387-2391.

40. Solomon TP, Haus JM, Kelly KR, et al. (2009) Randomized trial on the effects of a 7-d low-glycemic diet and exercise intervention on insulin resistance in older obese humans. Am J Clin Nutr 90, 1222-1229.

41. Pittas AG, Roberts SB, Das SK, et al. (2006) The effects of the dietary glycemic load on type 2 diabetes risk factors during weight loss. Obesity (Silver Spring) 14, 2200-2209.

42. Eckel RH (1992) Insulin resistance: an adaptation for weight maintenance. Lancet 340, 1452-1453.

43. Tremblay A, Boule N, Doucet E, et al. (2005) Is the insulin resistance syndrome the price to be paid to achieve body weight stability? Int J Obes (Lond) 29, 1295-1298.

44. Swinburn BA, Nyomba BL, Saad MF, et al. (1991) Insulin resistance associated with lower rates of weight gain in Pima Indians. J Clin Invest 88, 168-173.

45. Yamauchi T, Kamon J, Minokoshi Y, et al. (2002) Adiponectin stimulates glucose utilization and fatty-acid oxidation by activating AMP-activated protein kinase. Nat Med $\mathbf{8}$, $1288-1295$.

46. Astrand O, Carlsson M, Nilsson I, et al. (2010) Weight gain by hyperalimentation elevates C-reactive protein levels but does not affect circulating levels of adiponectin or resistin in healthy subjects. Eur J Endocrinol 163, 879-885.

47. Cook JR \& Semple RK (2010) Hypoadiponectinemia - cause or consequence of human "insulin resistance"? J Clin Endocrinol Metab 95, 1544-1554.

48. Weickert MO \& Pfeiffer AF (2008) Metabolic effects of dietary fiber consumption and prevention of diabetes. J Nutr $\mathbf{1 3 8}$, 439-442.

49. Andersson A, Tengblad S, Karlstrom B, et al. (2007) Wholegrain foods do not affect insulin sensitivity or markers of lipid peroxidation and inflammation in healthy, moderately overweight subjects. J Nutr 137, 1401-1407.

50. Galgani JE, Uauy RD, Aguirre CA, et al. (2008) Effect of the dietary fat quality on insulin sensitivity. Br J Nutr $\mathbf{1 0 0}$, $471-479$. 